\title{
The visible and near ultraviolet rotation-vibration spectrum of HOD
}

\author{
A. Jenouvrier and M.F. Mérienne \\ Spectrométrie Moléculaire et Atmosphérique, UFR Sciences, Moulin de la Housse, B.P. 1039, 51687 Reims Cedex 2, France \\ M. Carleer and R. Colin \\ Université Libre de Bruxelles, Service de Chimie Physique Moléculaire, CP 160/09, Av. F.D. Roosevelt 50, B-1050 Brussels, Belgium \\ E-mail: mcarleer@ulb.ac.be \\ A.-C. Vandaele \\ Institut d'Aeronomie Spatiale de Belgique, Av. Circulaire 3, B-1180 Brussels, Belgium \\ P.F. Bernath \\ Department of Chemistry, University of Waterloo, Waterloo, ON Canada N2L $3 G 1$ and Department of Chemistry, University of Arizona, Tucson, AZ, \\ 85721 \\ E-mail: bernath@uwaterloo.ca
}

Oleg L. Polyansky ${ }^{1}$ and Jonathan Tennyson

Department of Physics and Astronomy, University College London, London WC1E 6BT, UK

E-mail: j.tennyson@ucl.ac.uk

\begin{abstract}
A Fourier transform spectrum has been recorded of a $\mathrm{H}_{2} \mathrm{O} / \mathrm{D}_{2} \mathrm{O}$ vapor mixture in the wavenumber range 16,300 to $22,800 \mathrm{~cm}^{-1}$ using a long path cell. 410 lines of HDO are assigned to the OH stretching overtone bands $5 \nu_{3}, 6 \nu_{3}$ and $7 \nu_{3}$ plus combination bands. Assignments for the $6 \nu_{3}$ and $7 \nu_{3}$ bands represent the first data for the 006 and 007 states and give band origins of 19,836.88 $\mathrm{cm}^{-1}$ and $22,625.50 \mathrm{~cm}^{-1}$, respectively. Rotational term values for these states are also obtained.
\end{abstract}

\section{INTRODUCTION}

The visible and near UV overtone spectrum of water vapor is relatively well known from the classic work of Camy-Peyret et al. (1) as well as the more extensive recent work of Carleer et al. (2) and Zobov et al. (3). The main application of this work is in atmospheric science because water vapor is the primary absorber of sunlight.

Work on the HOD isotopomer is much less extensive. For example, the 005 and 015 states at $16,899 \mathrm{~cm}^{-1}$ and 18,208

\footnotetext{
1 Permanent address: Institute of Applied Physics, Russian Academy of Science, Uljanov Street 46, Nizhnii Novgorod, Russia 603024.
}

$\mathrm{cm}^{-1}$ (4-6) are the most highly-excited known vibrational energy levels as compared to the 800 level of $\mathrm{H}_{2} \mathrm{O}$ at $25,120 \mathrm{~cm}^{-1}$ (3). Note for HOD we adopt the traditional (but non-IUPAC) normal mode notation of $\nu_{1}$ as the OD stretching mode, $\nu_{2}$ as the bend and $\nu_{3}$ as the $\mathrm{OH}$ stretch.

Because $\mathrm{H}_{2} \mathrm{O}$ can have such a high abundance in the atmosphere, HOD is also an important atmospheric molecule. HOD can be readily observed in our atmosphere (7) and generally has a higher abundance than many well-known atmospheric molecules such as ozone. For example, if water has a mixing ratio of $1 \%$ (common in the troposphere) then HOD would have a volume mixing ratio of about $3 \mathrm{ppm}$, comparable to the important greenhouse gases $\mathrm{CH}_{4}$ and $\mathrm{N}_{2} \mathrm{O}$.

The infrared spectra of HOD were first analyzed by Benedict, Gailar and Plyler in 1956 (8). There has been much additional work since then, most notably by Toth and co-workers. The most recent and complete list of ground state energy levels is by Toth (9).

The visible and near infrared spectra of HOD have recently been studied by intracavity laser absorption spectroscopy in Grenoble. The 005 band (5) and the 015 band (6), the 004 band at $13,854 \mathrm{~cm}^{-1}(10), 023\left(\right.$ at $13,278 \mathrm{~cm}^{-1}$ ) and 103 (at 
$13,332 \mathrm{~cm}^{-1}$ ) (11), 014 (at 15,168 $\mathrm{cm}^{-1}$ ) (12) and 024 (at $16,456 \mathrm{~cm}^{-1}$ ) (6) were the primary bands studied. Hu et al. (13) have measured the 400 band near $12,767 \mathrm{~cm}^{-1}$ also by intracavity laser spectroscopy but they used a Fourier transform spectrometer rather than a spectrograph.

$\mathrm{HOD}$ differs from $\mathrm{H}_{2} \mathrm{O}$ in that the three vibrational modes have very different frequencies and there are fewer interactions between modes. In particular the strongest bands in the visible region are the overtones of the $\mathrm{OH}$ stretch and they behave like isolated states (5). This behavior has made HOD a popular molecule for bond-selective laser chemistry by dissociation (e.g., 14). The lines of the 004 band, for example, were first studied by photo-acoustic and photodissociation spectroscopy (14) and then with a pulsed optical parametric oscillator (15).

While intracavity absorption spectroscopy and other laserbased techniques have a very high sensitivity, direct long path absorption experiments with a Fourier transform spectrometer (FTS) offer a wide spectral coverage and a more precise wavenumber scale. We report here on our FTS measurements of the 005, 006 and 007 bands of HOD in the 16,300 - 22,800 $\mathrm{cm}^{-1}$ spectral region. There have been no previous measurements on HOD reported above $18,350 \mathrm{~cm}^{-1}$.

\section{EXPERIMENT}

The HOD and $\mathrm{H}_{2} \mathrm{O}$ experiments were carried out at the same time $(2,3)$. The measurements were made using the Bruker IFS $120 \mathrm{M}$ high resolution Fourier transform spectrometer of the Laboratoire de Chimie Physique Moléculaire (Université Libre de Bruxelles) coupled to the $50 \mathrm{~m}$ base length White-type multiple reflection absorption cell of the Groupe de Spectrométrie Moléculaire et Atmosphérique (Université de Reims). A 450 $\mathrm{W}$ xenon arc lamp was used as light source. Two detectors (a $\mathrm{Si}$ and a GaP diode) were used to record the spectral region from 10,000 to $30,000 \mathrm{~cm}^{-1}$. The spectra were recorded at room temperature $\left(18^{\circ} \mathrm{C}\right)$. We used 12 traversals through the cell, giving a total absorption path of $602.32 \mathrm{~m}$. This path was chosen because it maximizes the signal-to-noise $(\mathrm{S} / \mathrm{N})$ ratio of the recorded spectra.

A nominal mixture of $42.5 \% \mathrm{H}_{2} \mathrm{O}$ and $57.5 \% \mathrm{D}_{2} \mathrm{O}$ was introduced in the cell at a total pressure of 9.8 Torr. From the relative amounts of $\mathrm{H}_{2} \mathrm{O}$ and $\mathrm{D}_{2} \mathrm{O}$ added to the cell, we estimate that the proportions of $\mathrm{H}_{2} \mathrm{O} / \mathrm{HOD} / \mathrm{D}_{2} \mathrm{O}$ in the cell were about $19 \%, 47 \%$ and $34 \%$, respectively. The spectra were recorded at a resolution of $0.06 \mathrm{~cm}^{-1}$ (15 cm maximum optical path difference) in two overlapping pieces. In the visible-near infrared region $(13,100$ $-22,300 \mathrm{~cm}^{-1}$ ), the coaddition of some 2048 interferograms in a total recording time of the order of $12 \mathrm{~h}$ resulted in a $\mathrm{S} / \mathrm{N}$ ratio of 3000. In the blue-near ultraviolet part $(17,700-30,000$ $\mathrm{cm}^{-1}$ ) of the spectrum, the coaddition of 4096 interferograms ( $24 \mathrm{~h}$ of recording time) proved necessary to attain a $\mathrm{S} / \mathrm{N}$ ratio of 2500. A third region was recorded in the near infrared $(9,000$ $-15,000 \mathrm{~cm}^{-1}$ ) but the lines were mainly saturated and were not used.
Line positions and other parameters were measured in the near UV and visible spectra using a program called WSpectra developed by Carleer (16). Each line was fitted with a Voigt lineshape profile convolved by the instrumental function. After correction for the refractive index of air, the lines were calibrated by comparison with our previous water line positions $(2,3)$. Water lines were then removed from our linelists leaving a list of 1381 lines. Tests with spectra which were recorded at different $\mathrm{H}_{2} \mathrm{O} / \mathrm{D}_{2} \mathrm{O}$ concentrations suggest that there are no $\mathrm{D}_{2} \mathrm{O}$ lines in the $18,000-23,000 \mathrm{~cm}^{-1}$ region. The HOD line positions have an absolute accuracy of about $0.004 \mathrm{~cm}^{-1}$. The resulting linelist is available electronically.

\section{ANALYSIS}

Significant advances have recently been made in the analysis of short wavelength spectra of water by using variational calculations $(2,3)$. These sophisticated calculations are required for water because of the strong polyad structure and, in particular, the large number of possible mixings within the higher polyads which make the use of methods based on effective hamiltonians difficult to apply. The spectrum of HOD is somewhat different as the isotopic substitution breaks the polyad structure and leads to a much better separation between the vibrational modes. This means that for high-lying states the bright $\mathrm{OH}$ stretching overtones are well separated from other vibrational modes and can be analyzed in isolation.

Our initial analysis concentrated on assigning transitions to states of $v_{3}=5,6$ and 7 by extrapolating rotational constants from lower $v_{3}$ overtone states. This method allowed the assignment of many low $J$ transitions. During the course of this work Schwenke (17) updated the previous variational linelist for HOD (18). Use of this linelist confirmed many of the previous assignments and suggest some candidate transitions with higher rotational excitation. However, this linelist does not extend above $20,000 \mathrm{~cm}^{-1}$.

A second approach was then tested. First a computer program was used to identify all possible transitions between known energy levels ('trivial assignments'). For this purpose we used both our newly-determined energy levels, and those obtained previously $(5,6)$. Another program was then written which identified possible assignments based on combination differences. This produced a large number of possible assignments which were then reduced to a managable list of candidates using intensity considerations. Further assignments were made using the same procedure used to confirm the original assignments. However, many possible candidates could not be confirmed in this way and must await further work, probably using an improved linelist.

A total of 410 lines have been assigned to HDO including assignments obtained to the 015 and 024 combination bands. Approximately 150 of these assignments were taken from previous studies $(5,6)$. Tables 1 and 2 present our newly assigned transitions for the $6 \nu_{3}$ and $7 \nu_{3}$ bands, respectively. These tables also give relative intensities for the different transitions. These assignments, as well as those for the $5 \nu_{3}$ state and for the combination bands, are given as part of the electronic linelist. The 
assignments to 005 , which were largely performed prior to us becoming aware of the work of Bertseva et al. (5) and therefore independent of it, were found to be in complete agreement. This is probably a manifestation of the fact that HDO is, compared to water, a straightforward system with relatively few vibrational perturbations. Although Bertseva et al.'s coverage of the 005 band is greater than ours we still have some lines and energy levels not reported in that work including transitions to the $14_{114}$ rotational state.

Our new transitions can be combined with the term values obtained by Toth (9) to give energy levels for the vibrationallyexcited states. Table 3 presents our new levels for the $6 \nu_{3}$ and $7 \nu_{3}$ vibrational states. Levels whose energies have been obtained by more than one transition, as denoted by an $N$ value greater than one in the table, have been confirmed by combination differences. In practice we believe even the values for levels with $N=1$ are secure. Our energy level calculations show that the band origin for the $6 \nu_{3}$ state is at $19,836.88 \mathrm{~cm}^{-1}$ and that for the $7 \nu_{3}$ vibrational state is at $22,625.50 \mathrm{~cm}^{-1}$.

\section{CONCLUSIONS}

A long path length Fourier transform spectrum has been recorded of a $\mathrm{H}_{2} \mathrm{O} / \mathrm{D}_{2} \mathrm{O}$ mixture which yielded many lines of HDO in the $5 \nu_{3}, 6 \nu_{3}$ and $7 \nu_{3}$ vibrational bands plus combination bands. Assignments in the $6 \nu_{3}$ and $7 \nu_{3}$ bands represent the first data for these highly-excited $\mathrm{OH}$ stretching states.

The experimental study yielded 1381 lines over a $6000 \mathrm{~cm}^{-1}$ region. So far we have assigned 410 lines definitely to HDO, largely $\mathrm{OH}$ overtone stretching transitions. Further progress on the assignment of HDO lines in the spectra must await the calculation of a variational linelist valid over a more extended energy range. Such calculations are significantly more expensive than the corresponding calculations on water because of the loss of symmetry in HDO and also the increase in the number of states caused by deuteration. However the new data obtained here, particularly the vibrational band origins, form important input for spectroscopically-determined potentials which, in turn, can be used to compute extensive and reliable linelists. Work on this aspect of the problem is in progress at University College London.

\section{ACKNOWLEDGMENTS}

This work was supported by the Prime Minister's OfficeFederal Office for Scientific, Technical and Cultural Affairs and the Fonds National de la Recherche Scientifique (Belgium). Support was provided by the Centre National de Recherche Scientifique and the Institut National des Sciences de l'Univers through the Programme National de Chimie Atmospherique (contract 98N51/0324) (France). The work of O.L.P. was supported in part by the Russian Fund for Fundamental Studies. This work was supported by the Natural Sciences and Engineering Research Council of Canada (NSERC). Acknowledgment is made to the Petroleum Research Fund for partial support of this work. Some support was also provided by the NASA Labo- ratory Astrophysics Program, the UK Engineering and Science Research Council and the UK Natural Environment Research Council.

\section{REFERENCES}

1. C. Camy-Peyret, J.-M. Flaud, J.-Y. Mandin, J.-P. Chevillard, J. Brault, D.A. Ramsay, M. Vervloet and J. Chauville, J. Mol. Spectrosc. 113, 208-228 (1985).

2. M. Carleer, A. Jenouvrier, A.-C. Vandaele, P.F. Bernath, M.F. Mérienne, R. Colin, N.F. Zobov, O.L. Polyansky, J. Tennyson and V.A. Savin, J. Chem. Phys. 111, 2444-2450 (1999).

3. N.F. Zobov, D. Belmiloud, O.L. Polyansky, J. Tennyson, S.V. Shirin, M. Carleer, A. Jenouvrier, A.-C. Vandaele, P.F. Bernath, M.F. Mérienne and R. Colin, J. Chem. Phys. 113, 1546-1552 (2000).

4. A.D. Bykov, V.A. Kapitanov, O.V. Naumenko, T.M. Petrova, V.I. Serdyukov and L.N. Sinitsa, J. Mol. Spectrosc. 153, 197-207 (1992).

5. E. Bertseva, O. Naumenko and A. Campargue, J. Mol. Spectrosc. 203, 28-36 (2000).

6. A. Campargue, E. Bertseva and O. Naumenko, J. Mol. Spectrosc. 204, 94 105 (2000).

7. F.W. Irion, E.J. Moyer, M.R. Gunson, C.P. Rinsland, Y.L. Yung, H.A. Michelsen, R.J. Salawitch, A.Y. Chang, M.J. Newchurch, M.M. Abbas, M.C. Abrams and R. Zander, Geophys. Res. Lett. 23, 2381-2384 (1996).

8. W.S. Benedict, N. Gailar and E.K. Plyler, J. Chem. Phys. 24, 1139-1165 (1956).

9. R.A. Toth, J. Mol. Spectrosc. 195, 73-97 (1999).

10. O. Naumenko, E. Bertseva and A. Campargue, J. Mol. Spectrosc. 197, 122132 (1999).

11. O. Naumenko, E. Bertseva and A. Campargue, J. Mol. Spectrosc. 201, $297-$ 309 (2000).

12. O. Naumenko and A. Campargue, J. Mol. Spectrosc. 199, 59-72 (2000).

13. S. Hu, H. Lin, S. He, J. Cheng and Q. Zhu, Phys. Chem. Chem. Phys. 1, 3727-3730 (1999).

14. R.L. Vander Wal, J.L. Scott, F.F. Crim, K. Weide and R. Schinke, J. Chem. Phys. 94, 3548-3555 (1991).

15. J.R. Fair, O. Votava and D.J. Nesbitt, J. Chem. Phys. 108, 72-80 (1998).

16. M. Carleer, Remote Sensing of Clouds and the Atmosphere V, Proc. SPIE 4168, in press (2001).

17. D. W. Schwenke, http://george.arc.nasa.gov/ dschwenke/

18. H. Partridge and D. W. Schwenke, J. Chem. Phys. 106, 4618-4639 (1997). 
TABLE 1

\begin{tabular}{|c|c|c|c|c|c|c|c|}
\hline \multicolumn{2}{|c|}{$\begin{array}{c}\text { Assigned transitions } \\
\text { Intensities }\end{array}$} & \multicolumn{2}{|c|}{$\begin{array}{l}\text { in the } \\
\text { given }\end{array}$} & \multicolumn{2}{|c|}{$\begin{array}{c}6 \nu_{3} \quad \text { band } \\
\text { in } \quad \text { arbitrary } \\
\end{array}$} & \multicolumn{2}{|c|}{$\begin{array}{l}\text { of HDO. } \\
\text { units } \\
\end{array}$} \\
\hline$\nu / \mathrm{cm}^{-1}$ & Int & $J^{\prime}$ & $K_{a}^{\prime}$ & $K_{c}^{\prime}$ & $J^{\prime \prime}$ & $K_{a}^{\prime \prime}$ & $K_{c}^{\prime \prime}$ \\
\hline 19905.0276 & 0.0173 & 5 & 1 & 4 & 4 & 1 & 3 \\
\hline 19899.0979 & 0.0162 & 6 & 0 & 6 & 5 & 0 & 5 \\
\hline 19894.9758 & 0.0116 & 5 & 2 & 3 & 4 & 2 & 2 \\
\hline 19893.4305 & 0.0192 & 5 & 0 & 5 & 4 & 0 & 4 \\
\hline 19893.2688 & 0.0173 & 4 & 1 & 3 & 3 & 1 & 2 \\
\hline 19888.2104 & 0.0122 & 5 & 1 & 5 & 4 & 1 & 4 \\
\hline 19886.3719 & 0.0220 & 4 & 0 & 4 & 3 & 0 & 3 \\
\hline 19882.4303 & 0.0134 & 5 & 2 & 4 & 4 & 2 & 3 \\
\hline 19879.3349 & 0.0173 & 3 & 1 & 2 & 2 & 1 & 1 \\
\hline 19879.2615 & 0.0202 & 4 & 1 & 4 & 3 & 1 & 3 \\
\hline 19877.9379 & 0.0128 & 4 & 2 & 2 & 3 & 2 & 1 \\
\hline 19877.2228 & 0.0259 & 3 & 0 & 3 & 2 & 0 & 2 \\
\hline 19870.9360 & 0.0126 & 4 & 2 & 3 & 3 & 2 & 2 \\
\hline 19869.0122 & 0.0185 & 3 & 1 & 3 & 2 & 1 & 2 \\
\hline 19865.5737 & 0.0162 & 2 & 0 & 2 & 1 & 0 & 1 \\
\hline 19864.0450 & 0.0123 & 2 & 1 & 1 & 1 & 1 & 0 \\
\hline 19861.0568 & 0.0091 & 3 & 2 & 1 & 2 & 2 & 0 \\
\hline 19858.1202 & 0.0097 & 3 & 2 & 2 & 2 & 2 & 1 \\
\hline 19857.4805 & 0.0118 & 2 & 1 & 2 & 1 & 1 & 1 \\
\hline 19851.8494 & 0.0098 & 1 & 0 & 1 & 0 & 0 & 0 \\
\hline 19845.5662 & 0.0053 & 4 & 3 & 2 & 3 & 3 & 1 \\
\hline 19838.4211 & 0.0061 & 2 & 1 & 1 & 2 & 1 & 2 \\
\hline 19833.7071 & 0.0122 & 1 & 1 & 0 & 1 & 1 & 1 \\
\hline 19827.9329 & 0.0115 & 1 & 1 & 1 & 1 & 1 & 0 \\
\hline 19823.1772 & 0.0032 & 5 & 2 & 3 & 5 & 2 & 4 \\
\hline 19821.3695 & 0.0088 & 0 & 0 & 0 & 1 & 0 & 1 \\
\hline 19821.1100 & 0.0062 & 2 & 1 & 2 & 2 & 1 & 1 \\
\hline 19817.9723 & 0.0055 & 4 & 2 & 2 & 4 & 2 & 3 \\
\hline 19814.9192 & 0.0106 & 3 & 2 & 1 & 3 & 2 & 2 \\
\hline 19813.9643 & 0.0175 & 2 & 2 & 0 & 2 & 2 & 1 \\
\hline 19812.9285 & 0.0176 & 2 & 2 & 1 & 2 & 2 & 0 \\
\hline 19810.6848 & 0.0037 & 3 & 1 & 3 & 3 & 1 & 2 \\
\hline 19810.0101 & 0.0090 & 3 & 2 & 2 & 3 & 2 & 1 \\
\hline 19805.6792 & 0.0134 & 1 & 0 & 1 & 2 & 0 & 2 \\
\hline 19804.4783 & 0.0067 & 4 & 2 & 3 & 4 & 2 & 2 \\
\hline 19802.3121 & 0.0110 & 1 & 1 & 1 & 2 & 1 & 2 \\
\hline 19797.3332 & 0.0105 & 1 & 1 & 0 & 2 & 1 & 1 \\
\hline 19789.7509 & 0.0177 & 2 & 0 & 2 & 3 & 0 & 3 \\
\hline 19786.9005 & 0.0141 & 2 & 1 & 2 & 3 & 1 & 3 \\
\hline 19784.7329 & 0.0153 & 3 & 3 & 0 & 3 & 3 & 1 \\
\hline 19784.6106 & 0.0143 & 3 & 3 & 1 & 3 & 3 & 0 \\
\hline 19782.9099 & 0.0090 & 4 & 3 & 2 & 4 & 3 & 1 \\
\hline 19780.0863 & 0.0145 & 2 & 1 & 1 & 3 & 1 & 2 \\
\hline 19773.2313 & 0.0166 & 3 & 0 & 3 & 4 & 0 & 4 \\
\hline 19770.7577 & 0.0161 & 3 & 1 & 3 & 4 & 1 & 4 \\
\hline 19766.7580 & 0.0248 & 2 & 2 & 1 & 3 & 2 & 2 \\
\hline 19765.8269 & 0.0077 & 2 & 2 & 0 & 3 & 2 & 1 \\
\hline 19762.5422 & 0.0145 & 3 & 1 & 2 & 4 & 1 & 3 \\
\hline 19755.7567 & 0.0150 & 4 & 0 & 4 & 5 & 0 & 5 \\
\hline 19753.7888 & 0.0145 & 4 & 1 & 4 & 5 & 1 & 5 \\
\hline 19750.0327 & 0.0099 & 3 & 2 & 2 & 4 & 2 & 3 \\
\hline 19748.4875 & 0.0109 & 3 & 2 & 1 & 4 & 2 & 2 \\
\hline 19744.4954 & 0.0118 & 4 & 1 & 3 & 5 & 1 & 4 \\
\hline 19737.2698 & 0.0126 & 5 & 0 & 5 & 6 & 0 & 6 \\
\hline 19735.9791 & 0.0074 & 5 & 1 & 5 & 6 & 1 & 6 \\
\hline 19732.6874 & 0.0085 & 4 & 2 & 3 & 5 & 2 & 4 \\
\hline 19731.0133 & 0.0105 & 4 & 2 & 2 & 5 & 2 & 3 \\
\hline 19725.5045 & 0.0092 & 5 & 1 & 4 & 6 & 1 & 5 \\
\hline 19722.1149 & 0.0033 & 3 & 3 & 1 & 4 & 3 & 2 \\
\hline 19717.8861 & 0.0098 & 6 & 0 & 6 & 7 & 0 & 7 \\
\hline 19716.9998 & 0.0074 & 6 & 1 & 6 & 7 & 1 & 7 \\
\hline 19714.6006 & 0.0076 & 5 & 2 & 4 & 6 & 2 & 5 \\
\hline 19713.2623 & 0.0081 & 5 & 2 & 3 & 6 & 2 & 4 \\
\hline
\end{tabular}


TABLE 2

\begin{tabular}{|c|c|c|c|c|c|c|c|}
\hline \multicolumn{2}{|c|}{$\begin{array}{c}\text { Assigned transitions } \\
\text { Intensities are }\end{array}$} & \multicolumn{2}{|c|}{$\begin{array}{l}\text { in the } \\
\text { given }\end{array}$} & \multicolumn{2}{|c|}{$\begin{array}{cc}7 \nu_{3} & \text { band } \\
\text { in } & \text { arbitrary } \\
\end{array}$} & \multicolumn{2}{|c|}{$\begin{array}{l}\text { of HDO. } \\
\text { units }\end{array}$} \\
\hline$\nu / \mathrm{cm}^{-1}$ & Int & $J^{\prime}$ & $K_{a}^{\prime}$ & $K_{c}^{\prime}$ & $J^{\prime \prime}$ & $K_{a}^{\prime \prime}$ & $\overline{K_{c}^{\prime \prime}}$ \\
\hline 22679.6104 & 0.0022 & 5 & 0 & 5 & 4 & 0 & 4 \\
\hline 22674.0248 & 0.0018 & 5 & 1 & 5 & 4 & 1 & 4 \\
\hline 22673.4218 & 0.0022 & 4 & 0 & 4 & 3 & 0 & 3 \\
\hline 22666.7297 & 0.0016 & 3 & 1 & 2 & 2 & 1 & 1 \\
\hline 22665.9459 & 0.0023 & 4 & 1 & 4 & 3 & 1 & 3 \\
\hline 22665.0010 & 0.0025 & 3 & 0 & 3 & 2 & 0 & 2 \\
\hline 22656.2027 & 0.0021 & 3 & 1 & 3 & 2 & 1 & 2 \\
\hline 22653.8612 & 0.0019 & 2 & 0 & 2 & 1 & 0 & 1 \\
\hline 22640.3938 & 0.0014 & 1 & 0 & 1 & 0 & 0 & 0 \\
\hline 22621.5309 & 0.0013 & 1 & 1 & 0 & 1 & 1 & 1 \\
\hline 22615.7217 & 0.0014 & 1 & 1 & 1 & 1 & 1 & 0 \\
\hline 22609.9948 & 0.0015 & 0 & 0 & 0 & 1 & 0 & 1 \\
\hline 22599.4347 & 0.0018 & 2 & 2 & 0 & 2 & 2 & 1 \\
\hline 22598.3256 & 0.0020 & 2 & 2 & 1 & 2 & 2 & 0 \\
\hline 22594.2170 & 0.0017 & 1 & 0 & 1 & 2 & 0 & 2 \\
\hline 22590.0858 & 0.0018 & 1 & 1 & 1 & 2 & 1 & 2 \\
\hline 22585.1591 & 0.0014 & 1 & 1 & 0 & 2 & 1 & 1 \\
\hline 22578.0408 & 0.0023 & 2 & 0 & 2 & 3 & 0 & 3 \\
\hline 22576.1574 & 0.0024 & 2 & 1 & 2 & 3 & 1 & 3 \\
\hline 22566.2672 & 0.0016 & 3 & 3 & 0 & 3 & 3 & 1 \\
\hline 22566.1165 & 0.0017 & 3 & 3 & 1 & 3 & 3 & 0 \\
\hline 22561.0141 & 0.0022 & 3 & 0 & 3 & 4 & 0 & 4 \\
\hline 22557.9103 & 0.0000 & 3 & 1 & 3 & 4 & 1 & 4 \\
\hline 22551.3050 & 0.0009 & 2 & 2 & 0 & 3 & 2 & 1 \\
\hline 22549.9357 & 0.0018 & 3 & 1 & 2 & 4 & 1 & 3 \\
\hline 22542.8093 & 0.0015 & 4 & 0 & 4 & 5 & 0 & 5 \\
\hline 22540.4696 & 0.0020 & 4 & 1 & 4 & 5 & 1 & 5 \\
\hline 22523.4549 & 0.0018 & 5 & 0 & 5 & 6 & 0 & 6 \\
\hline 22521.7825 & 0.0013 & 5 & 1 & 5 & 6 & 1 & 6 \\
\hline 22503.6648 & 0.0009 & 3 & 3 & 1 & 4 & 3 & 2 \\
\hline
\end{tabular}

TABLE 3

HDO vibration-rotation energy levels in $\mathrm{cm}^{-1}$ derived using experimental data for the $6 \nu_{3}$ and $7 \nu_{3}$ vibrational states. ' $N$ ' gives the number of transitions observed to a particular level.

\begin{tabular}{|c|c|c|c|c|}
\hline$J K_{a} K_{c}$ & (006) & $\mathrm{N}$ & (007) & $\mathrm{N}$ \\
\hline 000 & 19836.8777 & 1 & 22625.5030 & 1 \\
\hline 101 & 19851.8509 & 2 & 22640.3919 & 2 \\
\hline 111 & 19860.4341 & 2 & 22648.2154 & 2 \\
\hline 110 & 19863.5167 & 2 & 22651.3416 & 2 \\
\hline 202 & 19881.0814 & 2 & 22669.3701 & 2 \\
\hline 212 & 19887.2916 & 3 & 22676.5482 & 1 \\
\hline 211 & 19896.5456 & 3 & & \\
\hline 221 & 19922.1723 & 2 & 22707.5946 & 1 \\
\hline 220 & 19922.8911 & 2 & 22708.3653 & 2 \\
\hline 303 & 19923.3916 & 2 & 22711.1721 & 2 \\
\hline 313 & 19927.1417 & 3 & 22714.3111 & 2 \\
\hline 312 & 19945.5225 & 2 & 22732.9166 & 2 \\
\hline 322 & 19967.0652 & 3 & & \\
\hline 321 & 19970.3192 & 3 & & \\
\hline 331 & 20017.6318 & 2 & 22799.1598 & 2 \\
\hline 330 & 20017.7565 & 1 & 22799.2908 & 1 \\
\hline 404 & 19977.7023 & 2 & 22764.7536 & 2 \\
\hline 414 & 19979.6529 & 2 & 22766.3355 & 2 \\
\hline 413 & 20009.7307 & 2 & & \\
\hline 423 & 20026.3211 & 3 & & \\
\hline 422 & 20035.0083 & 3 & & \\
\hline 432 & 20078.5885 & 2 & & \\
\hline 505 & 20043.5854 & 2 & 22829.7679 & 2 \\
\hline 515 & 20044.5936 & 2 & 22830.4025 & 2 \\
\hline 514 & 20088.0112 & 2 & & \\
\hline 524 & 20099.4740 & 2 & & \\
\hline 523 & 20116.8121 & 3 & & \\
\hline 606 & 20121.0456 & 2 & & \\
\hline
\end{tabular}

